\title{
Wie kommt es zum Jo-Jo-Effekt?
}

\begin{abstract}
Auch ein Jahr nach einer diätetisch bedingten Gewichtsabnahme sind die gegenregulatorischen Hormone der Appetitregulation, welche eine erneute Gewichtszunahme fördern, noch nicht auf das Niveau vor der Gewichtsabnahme zurückgekehrt. Diese Beobachtung eröffnet neue therapeutische Ansätze.
\end{abstract}

- Weltweit sind etwa 1,5 Milliarden Menschen übergewichtig, davon mindestens 400 Millionen adipös. In den Industrieländern versucht in der Zeiteinheit mindestens die Hälfte der übergewichtigen Bevölkerung über eine Nahrungsrestriktion das Gewicht zu reduzieren. Diese Bemühungen sind zunächst oft auch von Erfolg gekrönt, das Hauptproblem besteht bei den meisten Personen aber darin, dass nach kurzer Zeit das Ausgangsgewicht wieder erreicht und oft sogar überschritten wird.

Das Körpergewicht wird zentral reguliert, wobei zahlreiche hormonelle Signale aus dem Magen-Darm-Trakt, dem Pankreas und dem Fettgewebe an den Hypothalamus gesendet werden, wo Nahrungsaufnahme und Energieverbrauch gesteuert werden. Eine kalorische Restriktion führt zu akuten kompensatorischen Veränderungen mit Rückgang des Energieverbrauchs und der Konzentrationen von Leptin und Cholezystokinin bei gleichzeitigem Anstieg von Ghrelin, wodurch der Appetit gesteigert wird.

Es ist nicht bekannt, wie lange diese endokrinen Veränderungen bei Aufrechterhaltung des reduzierten Körpergewichts anhalten. 50 übergewichtige oder adipöse Patienten ohne Diabetes mellitus unterzogen sich einem zehnwöchigen Programm zur Gewichtsreduktion mit einer energiearmen Formeldiät von $500-550 \mathrm{kcal}$ pro Tag. Zu Beginn der Studie, in der 10. und in der 62. Woche wurden die Konzentrationen von Leptin, Ghrelin, Peptid YY, gastrischem inhibitorischen Polypeptid (GIP), Glukagon-like Peptid-1 (GLP-1), Amylin, pankreatischem Polypeptid (PP), Chole-
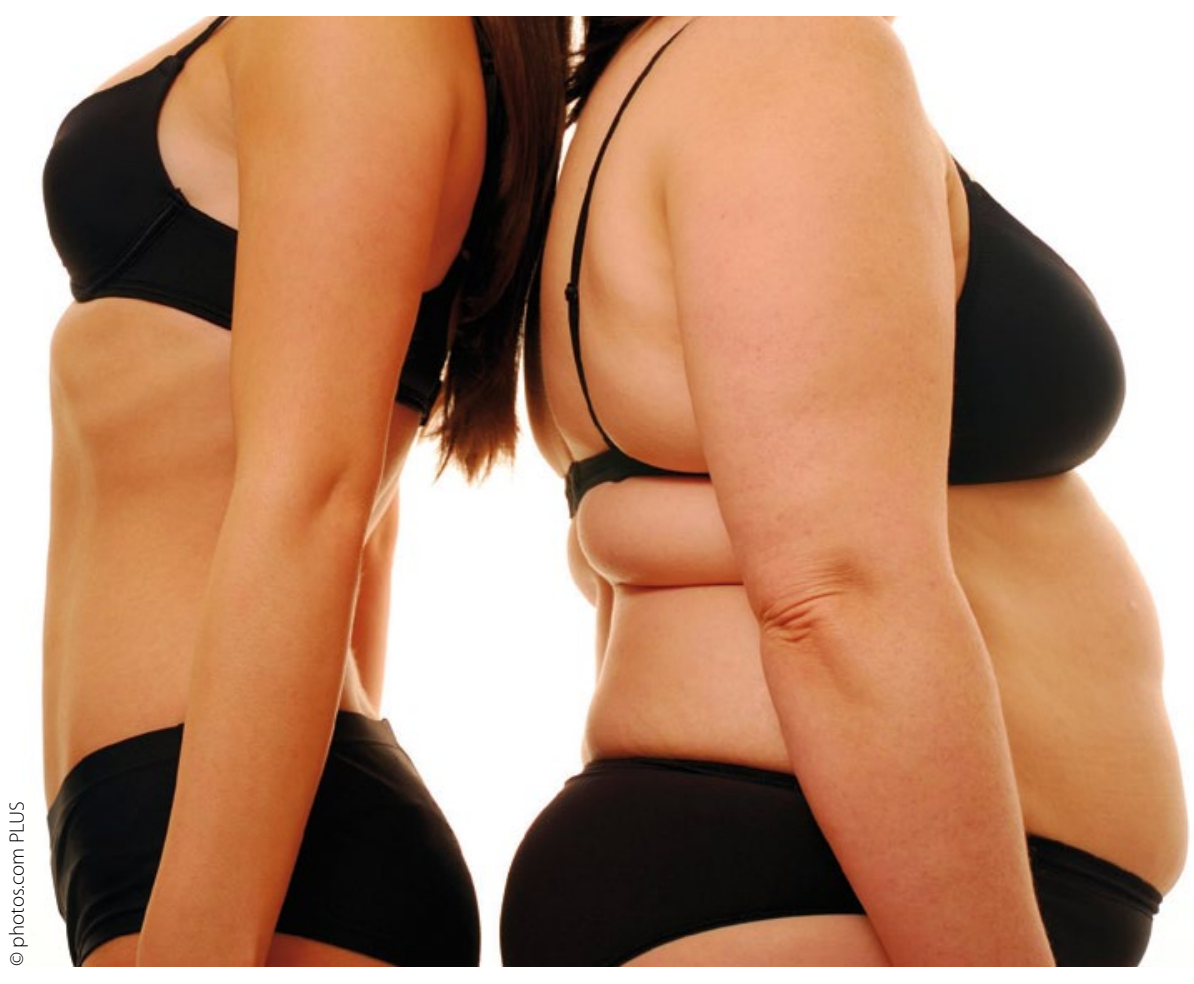

zystokinin (CCK) und Insulin bestimmt. Außerdem sollten die Probanden ihr Hungergefühl anhand einer visuellen Analogskala dokumentieren.

In der achten bis zehnten Woche kam es zu einem durchschnittlichen Gewichtsverlust von $13,5 \pm 0,5 \mathrm{~kg}(-14 \%$ des Ausgangsgewichts) und einer signifikanten Abnahme der Konzentrationen von Leptin, Peptid YY, CCK, Insulin und Amylin bei signifikanten Anstiegen von Ghrelin, GIP und PP. Erwartungsgemäß hatte auch der Appetit der Studienteilnehmer signifikant zugenommen. Nach einem Jahr war im Durchschnitt mehr als die Hälfte der Gewichtsreduktion wieder verschwunden. Dennoch bestanden immer noch signifikante Unterschiede in der Hormonkonstellation in ähnlicher Verteilung wie in der zehnten Woche. Auch das Hungergefühl war immer noch ausgeprägt.

\section{- P. Sumithran et al.}

(Korres.: Dr. Proietto, University o Melbourne, Department of Medicine, Heidelberg Repatriation Hospital, 300 Waterdale Rd., Heidelberg, VIC 3081, Australia): Long-term persistence of hormonal adaptations to weight loss. New Engl. J. Med. 365 (2011) 17, 1597-1604.
Sind Hormone schuld, wenn eine Gewichtsreduktion nicht von Dauer ist?

Kommentar

Der bei den meisten adipösen Personen unter hypokalorisch induzierter Gewichtsreduktion beobachtete sog. Jo-Jo-Effekt beruht also keinesfalls auf der bloßen willentlichen Wiederaufnahme alter Gewohnheiten, sondern hat einen ausgeprägten endokrinen Hintergrund. Die gegenregulatorische Auslenkung der Hormone, welche für eine höhere Energieaufnahme bzw. verringerten Energieverlust verantwortlich sind, bleibt über mindestens zwölf Monate hinweg erhalten und zwar auch dann noch, wenn das ursprünglich vorhandene Körpergewicht fast wieder erreicht ist. Aufgrund dieser Mechanismen ist es fast unmöglich, das durch Kalorienrestriktion erreichte niedrigere Körpergewicht dauerhaft zu halten. Zukünftige Bemühungen pharmakologischer Art werden diese Beobachtungen mit in ihr Kalkül einbeziehen müssen.

H. S. FÜESSL = 